\title{
Binding of Biotin to Streptavidin: A combined fluorescence correlation spectroscopy and time-resolved fluorescence study
}

\author{
J. Strömqvist ${ }^{1}$, L. Nardo ${ }^{2, a}$, O. Broekmans ${ }^{3}$, J. Kohn ${ }^{4}$, M. Lamperti ${ }^{2}$,

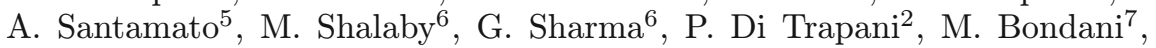 \\ and R. Rigler ${ }^{8,9}$ \\ ${ }^{1}$ Department of Applied Physics, Kungliga Tekniska Högskolan, 10044 Stockholm, \\ Sweden \\ 2 Dipartimento di Fisica e Matematica, Università degli Studi dell'Insubria and C.N.I.S.M., \\ via Valleggio 11, 22100 Como, Italy \\ 3 Department of Physics and Astronomy, Vrije Universiteit Amsterdam, De Boelelaan 1081, \\ 1081 HV Amsterdam, The Netherlands \\ 4 Department of Physics, University of Fribourg, Chemin du Musée 3, 1700 Fribourg, \\ Switzerland \\ ${ }^{5}$ Centre for Quantum Photonics, H. H. Wills Physics Laboratory and Department of \\ Electrical and Electronic Engineering, University of Bristol, Bristol, UK \\ ${ }^{6}$ INRS-EMT Université du Québec, 1650 Blvd. Lionel Boulet, Varennes, Quebec J3X 1S2, \\ Canada \\ 7 Istituto di Fotonica e Nanotecnologie, Consiglio Nazionale delle Ricerche and C.N.I.S.M., \\ via Valleggio 11, 22100 Como, Italy \\ 8 Department of Medical Biophysics, Karolinska Institutet, 17177 Stockholm, Sweden \\ 9 Laboratory of Biomedical Optics, Swiss Federal Institute of Technology, 1015 Lausanne, \\ Switzerland
}

\begin{abstract}
The Biotin-Streptavidin complex is a widely studied system in biology and biophysics, because of its extremely strong non-covalent binding affinity. The latter is often exploited to link molecules to substrates or to one another. However, the details of the BiotinStreptavidin binding have not been fully elucidated so far. Particularly, the role of cooperative effects in enhancing the binding affinity has not been clarified. Our long-term aim is to investigate this point by implementing two complementary approaches, fluorescence correlation spectroscopy and time-correlated single-photon counting. As both methods rely on the analysis of fluorescence signals, biotin labeled with Atto-550-dye was used. In this work, in order to get a first overview of the system, we analyzed solutions in three paradigmatic ranges of Biotin-to-Streptavidin concentration ratio. Fluorescence correlation spectroscopy measurements allowed us to extract diffusion times of free biotin and of biotin-Streptavidin complexes, and also to gain
\end{abstract}

${ }^{a}$ e-mail: luca.nardo@uninsubria.it 


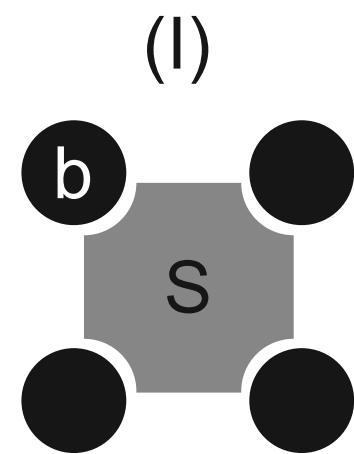

(II)

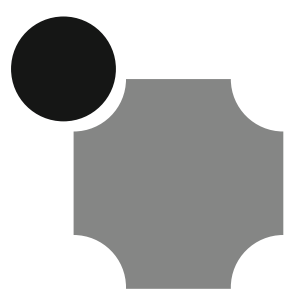

(III)

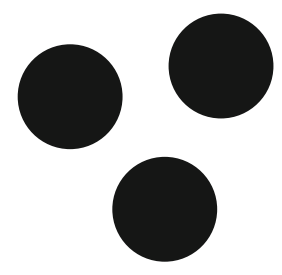

Fig. 1. The three species that are assumed to be present in the samples. (I) Saturated Streptavidin (s), with four bound Biotin (b) molecules; (II) marginally bound Streptavidin; (III) free Biotin.

\begin{abstract}
information about the dynamics of the intersystem crossing between the first excited triplet and the first excited singlet states. Timecorrelated single-photon counting made it possible to derive the lifetimes of the different species in solution, as well as to deduce relevant information about the relative abundance of Streptavidin-complexed and free Biotin.
\end{abstract}

\title{
1 Introduction
}

The interaction between Biotin and Streptavidin belongs to the strongest known today in bioscience. For this reason, it is of great interest to elucidate the background and the forces behind this interaction. The high value of the binding constant, $K_{d} \sim 10^{-14} \mathrm{M}[1]$, allows characterizing the Biotin-Streptavidin interaction at nanomolar concentrations and has been used as "searching tool" for other processes linked to the Biotin-Streptavidin system. Moreover, the Biotin-Streptavidin affinity has been widely exploited in biophysics to fix biomolecules to a substrate, e.g. in molecular tweezers or atomic force microscopy studies [2-5].

Streptavidin is a tetrameric protein in which each of the monomers hosts a binding site for Biotin (fig. 1). The binding of Biotin to Streptavidin is known to induce conformational changes in the protein structure that in turn notably enhance the thermodynamic stability of the quaternary structure [6]. The extremely high stability against dissociation of the saturated (4:1) Biotin-Streptavidin complex, as well as the high binding affinity, have been interpreted by suggesting a cooperative binding model in which the allosteric transition induced in the protein structure by the binding of the first Biotin molecule drives the remaining binding sites to saturation [7]. However, other authors report evidence in favor of non-cooperative binding [8]. Briefly, in spite of the relevance of this biochemical reaction, its details have not been fully understood yet [9].

For this reason we have started an analysis of the interactions between Biotin and Streptavidin using the tool of fluorescence correlation spectroscopy (FCS), which is able to provide quantitative information on the interactions between Streptavidin and fluorescently labeled Biotin in concentrations between 1 to 10 molecules per detected volume element and below. In the present work, the binding of Atto-550-labeled Biotin to Streptavidin has been preliminarily probed by FCS. Namely, three extreme situations have been analyzed: free Biotin; a Streptavidin-Biotin mixture bearing a strong excess of Streptavidin (1:10 Biotin-to-Strepavidin concentration ratio); and 
one bearing an excess of Biotin (10:1). The singlet-triplet intersystem crossing of Atto-550 as well as the diffusion kinetics of both the free Biotin and the BiotinStreptavidin complexes were elucidated. A parallel time-resolved fluorescence analysis based on time-correlated single-photon counting (TCSPC) measurements has also been undertaken. A combined analysis of FCS and TCSPC data allowed us to estimate the fractions of bound and free chromophore in the mixture bearing excess Biotin. The observation of substantial fluorescence quenching in the latter solution was interpreted as saturation of Streptavidin binding sites, with consequent self-quenching of Atto-550 by the fluorescence resonance energy transfer (FRET) mechanism.

These preliminary results represent a first step in our attempt to fully characterize the Biotin-Streptavidin binding equilibrium. In particular, details of the interaction such as the number of Biotin molecules bound to each Streptavidin, the cooperative or non-cooperative character of the binding and the binding kinetics will be addressed on the basis of the present results.

\section{Theoretical background}

\subsection{Fluorescence correlation spectroscopy}

Fluorescence correlation spectroscopy (FCS) consists in a statistical analysis of the temporal fluctuations of the fluorescence intensity emitted by samples of small numbers of fluorophores. Such fluctuations can be caused by e.g. small fluctuations in the sample concentration, or, more generally, by fluctuations in the thermodynamic parameters of the system [10-12]. Since such fluctuations are of the order of the square root of the average number of molecules composing the sample, they are negligibly small in the specimens typically investigated by conventional fluorescence analysis. The selection of the fluorescence signal emitted by only a very small number of molecules is most conveniently pursued by recurring to a confocal excitation/detection setup. The confocal configuration, which is fully described elsewhere [10-12], relies on excitation in a small, dual-conical volume inside the specimen, through diffraction-limited focusing of the excitation beam by means of a high numerical aperture immersion objective. Only the fluorescence coming from the Rayleigh range is then collected in epifluorescence configuration by coupling it into a spatial filtering optical system. Such a system consists of a telescope with a pin-hole put in the common focus of the entrance and exit lenses.

We introduce the temporal correlation function:

$$
G(\tau)=\langle\delta F(t) \delta F(t+\tau)\rangle /\langle F(t)\rangle^{2},
$$

where $\delta F(t)$ is the intensity fluctuation around the mean value. The analysis of the correlation function allows deriving local concentrations, mobility coefficients, intersystem crossing rates, and rate constants of chemical reactions of fluorophores or fluorescently labeled reactants, notably including biomolecules. The FCS approach displays several advantages over ensemble techniques, as it combines maximum sensitivity with high statistical confidence. Moreover, it enables working at minimal concentrations of sample, which is often mandatory in biophysics. In the following we will derive the analytical form of the autocorrelation function in two very simple instances, namely:

1. when the only source of fluorescence fluctuations is diffusion of fluorophores in and out of the confocal volume, with consequent changes in the local concentration; 
2. when also intersystem crossing from the fluorescent first excited singlet state $S_{1}$ of the fluorophores to the dark triplet state $T_{1}$ laying between $S_{1}$ and the ground state $S_{0}$ and back takes place.

The latter is a very common excited state mechanism for high quantum-yield dyes.

In doing so, we will make the following assumptions:

i. the background intensity is negligible;

ii. the expectation value of the fluorescence signal $\langle F(t)\rangle$ keeps constant within the acquisition of the correlation function;

iii. the confocal observation volume has a 3D Gaussian shape, that is:

$$
V_{\text {obs }} \propto \exp \left(-2\left(x^{2}+y^{2}\right) / r_{0}^{2}\right) \exp \left(-2 z^{2} / z_{0}^{2}\right) .
$$

In case 1., if only one species of fluorophores is present in the specimen, $\delta F$ can be written as

$$
\delta F=\sigma \Phi \kappa V_{o b s} \delta C=\eta V_{o b s} \delta C,
$$

where $\delta C$ represents the fluctuations in fluorophore concentration, $\sigma$ is the molecular absorption cross section of the fluorophore, $\Phi$ its fluorescence quantum yield, and $\kappa$ the overall detection quantum efficiency of the system. The parameter $\eta$ thus represents the molecular brightness of the diffusing species. In case the molecules are free to diffuse randomly and the total specimen volume is much greater than $V_{o b s}$, evaluating $G$ gives:

$$
G(\tau)=V_{\text {eff }}^{-1}\langle C\rangle^{-1}\left(1+\tau / \tau_{d}\right)^{-1}\left(1+r_{0}^{2} \tau / z_{0}^{2} \tau_{d}\right)^{-1 / 2},
$$

where $\tau_{d}$, the diffusion time of the fluorophore, is given by

$$
\tau_{d}=r_{0}^{2} / 4 D \text {. }
$$

The quantity $V_{\text {eff }}$ in Eq. (4) is no more the real observation volume. It is called the effective measurement volume element and is given by the formula

$$
V_{e f f}=\frac{\left(\int V_{o b s}(\mathbf{r}) \mathrm{d} V\right)^{2}}{\int V_{o b s}^{2}(\mathbf{r}) \mathrm{d} V} .
$$

Evaluating the above integrals over the whole space yields

$$
V_{\text {eff }}=\pi^{3 / 2} r_{0}^{2} z_{0} .
$$

Note that the value of $G(\tau)$ for $\tau \rightarrow 0$ is

$$
G(0)=\left(V_{e f f}\langle C\rangle\right)^{-1},
$$

which can be interpreted as the inverse of the average number of molecules in the effective measurement volume element and is bound to the absolute local concentration in the observation volume. In the case that more than one species of fluorophores are present, each of which is diffusing with a characteristic time $\tau_{d, i}$, Eq. (4) becomes

$$
G(\tau)=V_{\text {eff }}^{-1} \sum_{n} \eta_{n}\left\langle C_{n}\right\rangle\left(1+\frac{\tau}{\tau_{d}, n}\right)^{-1}\left(1+\frac{r_{0}^{2} \tau}{z_{0}^{2} \tau_{d, n}}\right)^{-1 / 2} /\left(\sum_{n} \eta_{n}\left\langle C_{n}\right\rangle\right)^{2},
$$

where we have taken into account that also the molecular brightnesses of the various diffusing species, which appear in Eq. (3), can be in principle different. 
Let's now consider case 2., that takes into account the effects of $S_{1} \leftrightarrow T_{1}$ interconversion dynamics. The latter reduce, from a phenomenological standpoint, to the occurrence of short dark intervals interrupting the bursts of fluorescence associated to the passage of fluorophore molecules within the observation volume. The correlation curve exhibits in this case an additional short-correlation-time shoulder, which can be modelled by means of an exponentially decaying term multiplying the diffusional component. Namely,

$$
G(\tau)=G_{d i f f}(\tau) G_{t r i p l}(\tau)=G_{d i f f}(\tau)\left(1-T+T \exp \left(-\tau / \tau_{T}\right)\right) /(1-T),
$$

where $G_{\text {diff }}$ is the purely diffusional correlation function of Eq. (4), $G_{\text {tripl }}$ is the new term due to $S_{1} \leftrightarrow T_{1}$ interconversion and depends from the two parameters $\tau_{T}$ and $T$, representing the characteristic interconversion time and the fraction of molecules undergoing intersystem dynamics, respectively.

\subsection{Time-correlated single photon counting}

Two complementary classes of techniques allow assessment of the parameters of a fluorescence decay (amplitudes and decay times): the frequency domain techniques and the time domain techniques. In the case of measurements in the frequency domain, the fluorescent sample is excited by a source of light whose intensity varies in time with a high frequency sinusoidal modulation. The intensity of the fluorescence signal emitted by the sample oscillates with the same frequency of the exciting beam, but it is phase-shifted and partially demodulated with respect to excitation: by measuring phase-shift and demodulation, frequency domain techniques yield the fluorescence decay time of the sample. Moreover, they are also capable to assess the single exponential or multi exponential nature of a decay by subsequent excitation of the sample with light modulated over a range of frequencies (typically ten or more) because of the intrinsic limitations of resolving the components of a multi-exponential decay using only one frequency. In time domain techniques a short pulse of light is made to impinge on the sample, and the temporal distribution of the photons re-emerging from the sample is directly recorded. The time and the frequency domain techniques theoretically yield equivalent data. However, in the case of multiple decay components, frequency domain techniques yield the relative fluorescence intensities $\alpha_{i}$. Conversely, in the very frequent case in which the different decay components are associated to different states of the same fluorophore (e.g. when multiple structural isomers are possible or when the fluorophore can be surrounded by different microenvironments), time-resolved methods allow straightforward determination of the fraction of fluorophores decaying with each lifetime. Indeed, for a given experimental fluorescence decay pattern

$$
F(t)=\sum_{i} A_{i} \exp \left(-\frac{t}{\tau_{i}}\right)
$$

the total fluorescence intensity detected during the measurement is

$$
F_{T}=\int_{0}^{\infty} d t F(t)
$$

The latter is equal to

$$
F_{T}=\sum_{i} \kappa \phi_{i} N_{i}
$$

where $N_{i}$ is the total number of molecules of species $i$ excited during the measurement and $\phi_{i}$ is the fluorescence quantum yield of species $i$. The proportionality factor 
$\kappa$ is called the detection quantum efficiency and depends on the fluorescence collection/detection apparatus only. However, it should be noted that it is slightly wavelength dependent, thus it is not strictly equal from a species to another in case the emission spectra of the two species are significantly different. Let us now consider the contribution to the detected fluorescence due to species $i$. This is given by

$$
F_{i}=\kappa \phi_{i} N_{i}=\int_{0}^{\infty} d t A_{i} \exp \left(-\frac{t}{\tau_{i}}\right) .
$$

The relative fluorescence intensities are defined as $\alpha_{i}=F_{i} / F_{T}$, and the fraction, $f_{i}$, of the fluorophores emitting with lifetime $\tau_{i}$, is

$$
f_{i}=\frac{N_{i}}{\sum_{i} N_{i}} .
$$

We now remind that

$$
\phi_{i}=\frac{k_{R, i}}{k_{R, i}+k_{N R, i}}
$$

and

$$
\tau_{i}=\frac{1}{k_{R, i}+k_{N R, i}},
$$

where $k_{R, i}$ and $k_{N R, i}$ are the radiative and non-radiative decay rate of species $i$, respectively. If the species $i$ represent different states of the same fluorophore, $k_{R, i}=$ $k_{R} \forall i$. By calculating the integrals in Eqs. (12) and (14) $\left(F_{T}=\sum_{i} A_{i} \tau_{i}\right.$ and $\left.F_{i}=A_{i} \tau_{i}\right)$ we obtain

$$
A_{i}=\frac{F_{i}}{\tau_{i}}=\frac{\kappa \phi_{i} N_{i}}{\tau_{i}}=\kappa k_{R} N_{i}
$$

that must be divided by the initial amplitude of the fluorescence decay distribution

$$
F(0)=\sum_{i} A_{i}=\kappa k_{R} \sum_{i} N_{i}
$$

to yield $f_{i}$. Conceptually, the time domain profile of a light pulse could be directly acquired by means of single photon counting (SPC) detectors by building a histogram of the number of photons detected in successive sampling time intervals much shorter than the pulse duration. Unfortunately, the light signals relevant to biomedical optics studies typically have pulse durations of few nanoseconds or less. Thus, sampling time interval widths in the picoseconds range would have to be used to obtain decent profiles. Consequently, "count and store" data acquisition electronics operating at the unconceivable rate of several terahertz would be needed. However, in biomedical applications it is frequently relevant to study the properties of a great number of virtually equal pulses, belonging to a periodic pulse train. In such a case, the time profile of a typical pulse can be very effectively recovered by applying the reconstruction technique called TCSPC, which, although based on SPC, allows to overcome the operational limits of the detection/storing electronics, by approaching the data acquisition process in a way slightly different from "count and store" methods, namely:

1. for each pulse of the pulse train to be analyzed, at most one photon must be detected

2. the arrival of each pulse of the train must be heralded by a signal triggering the timing device

3. the time elapsing from the heralding signal to detection of the photon (lag time) must be measured 


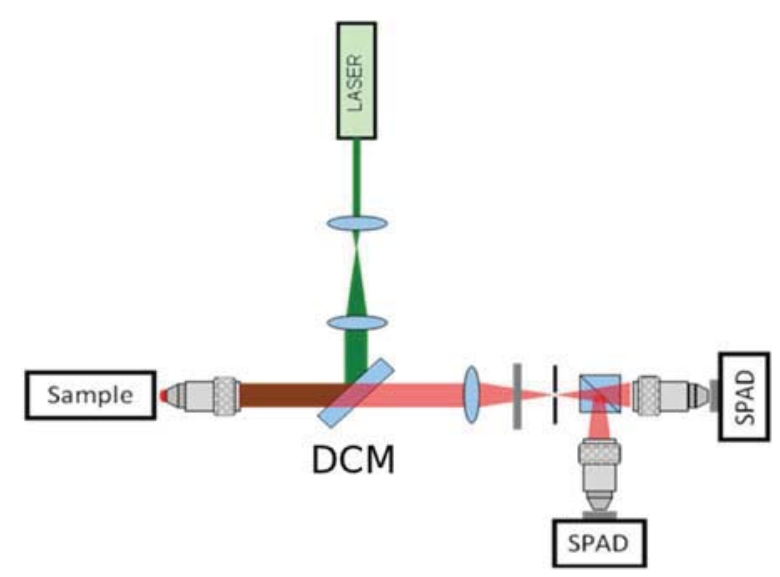

Fig. 2. Fluorescence correlation spectroscopy setup.

4. the lag times must be digitalized into sampling intervals and the photons being detected within each lag interval must be counted.

It is worth noting that the output data of TCSPC are histograms of the number of detected photons versus time analogous to those that would be ideally obtained by applying a "count and store" procedure.

The TCSPC pulse reconstruction procedure can be schematized as follows. The detector signal consists of a train of randomly distributed pulses due to the detection of individual photons. When a photon is detected, the time of the corresponding detector pulse is measured. The events are collected in order to build up the histogram of the detection times approximating the waveform of the optical pulse. It should be noted that the detection/timing/storing electronics cannot reset within few nanoseconds. Thus, only one photon per pulse can be timed. Thus, it is very important that the probability that more than one photon per excitation pulse impinge on the detector sensitive area be negligible. Otherwise, only the least-lagged photon data would be acquired, and the pulse temporal profile would be distorted.

\section{Experimental setup}

\subsection{Samples and sample preparation}

Biotin-Atto-550 (MW 1004.7) conjugate and Streptavidin (MW 52,800) were obtained from Sigma Aldrich. All dilutions were made in $50 \mathrm{mM}$ phosphate buffered saline (PBS) at $\mathrm{pH}$ 7.4.

We studied three samples: free Biotin (no Streptavidin); an excess of Streptavidin with respect to Biotin; and an excess of Biotin with respect to Streptavidin. Across all samples, the Biotin concentration was kept constant at $100 \mathrm{nM}$.

\subsection{Fluorescence correlation spectroscopy measurements}

\section{FCS Setup}

The FCS measurements were performed by using an assembled apparatus designed and built during the school. The system is sketched in Fig. 2. The excitation source 
was a CW diode laser emitting $30 \mathrm{~mW}$ of $532 \mathrm{~nm}$ light (Crisel Instruments, Roma, IT), suitably attenuated by means of neutral density filters in order to get $\mathrm{a} \approx 1 \mathrm{~mW}$ power impinging on the sample. The size of the laser spot was magnified by a factor of 3 using a telescopic system. The beam was sent to the water-immersion objective $(40 \times, 1.25 \mathrm{NA}$, Zeiss) by using a dichroic mirror (DCM in Fig. 2). A droplet $(50 \mu \mathrm{l})$ of the sample solution was put on a microscope cover glass of $170 \mu \mathrm{m}$ thickness, and placed on top of the objective. The fluorescence collected by the objective was then transmitted by the DCM and focused by another lens onto a $30 \mu \mathrm{m}$ diameter pinhole. The position of the pinhole was critical, because it needed to be placed exactly at the focal point of the lens, in order to block any out-of-focus fluorescence collected by the objective (confocal principle, see Sect. 2.2). As the fluorescence wavelength (576 nm [13]) was close to the excitation wavelength, a $550 \mathrm{~nm}$ cutoff filter (LL 550, Corion, Holliston, MA) was used to prevent the residual excitation stray light reaching the detectors. The fluorescence signal was processed using a cross-correlation approach. Namely, a $50 \%$ beam splitting cube divided the beam into two parts which were sent to the detectors, two single-photon avalanche diodes (SPADs) with builtin active quenching circuit and refrigeration system (PDM50 Micro-photon-devices, Bolzano, IT). Unlike conventional FCS systems, where only one photodiode is used, cross-correlation FCS allowed us to remove the fictitious contributions introduced in the correlation function by detector afterpulsing and dead time. The cross correlation functions were calculated in real time by a two-channel correlator (Flex2k-12x2, Correlator.com, Bridgewater, NJ).

\section{FCS data analysis}

The correlation curves obtained from the FCS measurements were fitted to the following expression:

$$
\begin{aligned}
G(\tau)= & +\frac{1}{N^{2}}\left[N m\left(1+\frac{\tau}{\tau_{B}}\right)^{-1}\left(1+\frac{\tau}{s^{2} \tau_{B}}\right)^{-1 / 2}+\right. \\
& \left.+N(1-m)\left(1+\frac{\tau}{\tau_{S}}\right)^{-1}\left(1+\frac{\tau}{s^{2} \tau_{S}}\right)^{-1 / 2}\right] \frac{1-T-T \exp \left(\tau / \tau_{T}\right)}{1-T}
\end{aligned}
$$

This formula can be easily derived from Eq. (9), for $n=2$. We made the further assumption that the molecular brightness of our species I, II and III were comparable and we set $\eta_{i}=1$. Here, $m$ is the fraction of free Biotin molecules, $N$ is the total average number of Biotin molecules (either free or bound to Streptavidin) in $V_{e f f}$, $\tau_{B}$ is the diffusion time of free Biotin, $\tau_{S}$ that of Streptavidin, and $s$ is the ratio of the longitudinal to the radial dimension of the laser focal spot, $r_{0} / z_{0}$ (see Eq. (9)) and has been fixed to the value $s=5$. The same expression has been used to fit the experimental data to a single diffusional component, by setting $m$ to either 1 (for pure Biotin) or 0 (for excess Streptavidin).

In Fig. 1, the three species that are assumed to be present in our samples are sketched. In the case of a sample of free Biotin, only species III is present; while in excess of Streptavidin with respect to Biotin, we expect species II to be dominant. For this reason, we fitted the corresponding correlation functions to the single-species model. With the above assumptions, the free Biotin and Streptavidin diffusion times $\left(\tau_{B}\right.$ and $\left.\tau_{S}\right)$ could be extracted, and used to initialize the two-species fit of the data obtained for the sample with an excess of Biotin. For this sample, it is most probable that either saturated Streptavidin (I) or free Biotin (III) are present. However, the masses, and thus the diffusion coefficients, of species I and II are very similar. This 


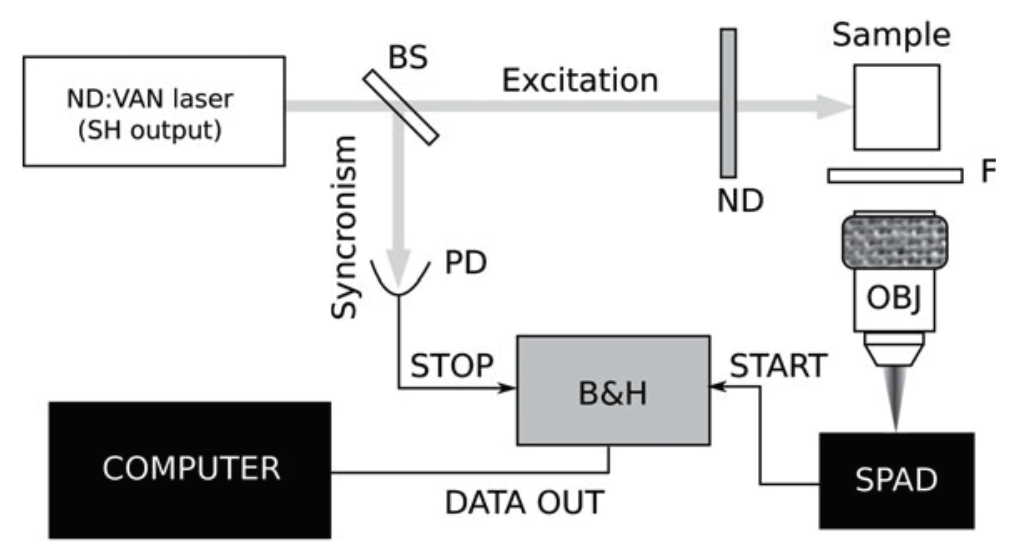

Fig. 3. Time-correlated single photon counting setup.

justifies without any further assumption the use of the two-species model in this case. In this work, we actually fixed the diffusion times of Streptavidin $\left(\tau_{S}\right)$ and Biotin $\left(\tau_{B}\right)$ to the values found with the one-species model.

\subsection{Time-correlated single photon counting measurements}

Time-correlated single-photon counting setup

The time-resolved fluorescence measurements were performed on the same kind of samples used in the FCS measurement described above. We used a time-correlated single-photon counting (TCSPC) system which is described in details elsewhere [14] and sketched in Fig. 3. Briefly, a pulsed $532 \mathrm{~nm} \mathrm{Nd:VAN} \mathrm{laser} \mathrm{(GE-100,} \mathrm{Time} \mathrm{band-}$ width product, Zurich, $\mathrm{CH}$ ) delivering pulses of $6 \mathrm{ps}$ duration at a repetition rate of $113 \mathrm{MHz}$ has been used. The fluorescence was collected at an angle of $90^{\circ}$ with respect to the incoming excitation beam. For the detection, one SPAD detector of the same type as those of the FCS setup has been used. The data were acquired by means of an integrated TCSPC PC board (SPC 150, Becker \& Hickl, Berlin, DE), which is run in reversed start-stop configuration [15] to minimize the dead time of the time-to-amplitude converter. The overall instrumental response function of the apparatus has $<30$ ps FWHM.

\section{TCSPC data analysis}

The experimental fluorescence decay patterns were fitted to multiexponential decays above a constant background accounting for non-time-correlated noise and detector dark counts, according to the equation:

$$
I(t)=I_{0}+\sum_{i} A_{i} \exp \left(\left(\tau_{0}-t\right) / \tau_{i}\right)
$$

where $I_{0}$ is the background intensity, the $A_{i}$ are the initial amplitudes of the decay components corresponding to the different species, $\tau_{0}$ is the arrival time of the excitation laser pulses, which has been kept fixed to the peak of the decay histogram during the fitting procedure, $\tau_{i}$ represent the lifetimes of either species I, II or III. The number of decay components was selected by adding components one by one until further additions did not bring about improvements in the $\chi^{2}$ value. 


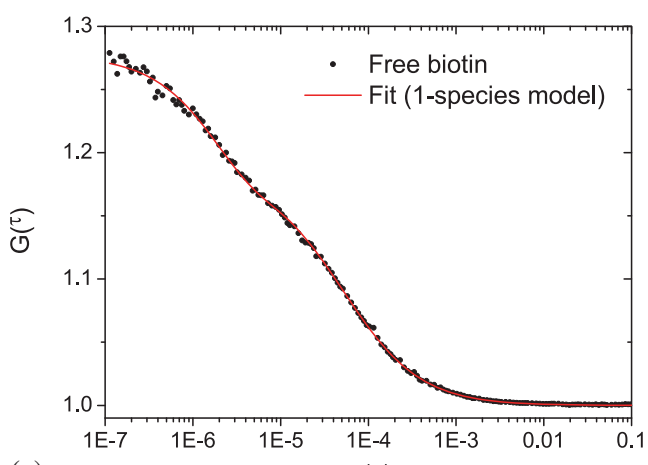

(a)

$\tau(\mathrm{s})$

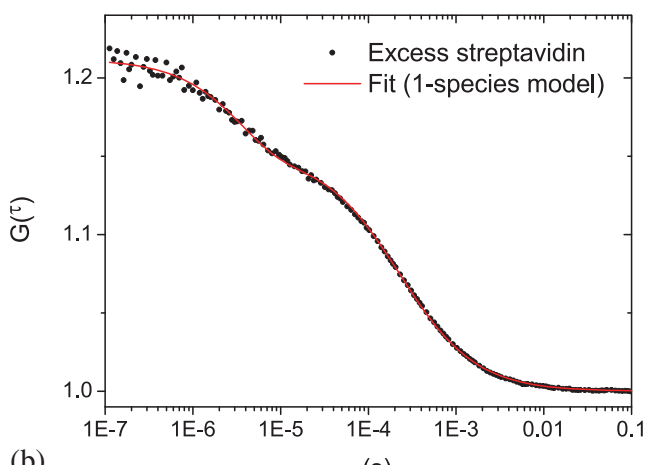

(b)

$\tau(\mathrm{s})$

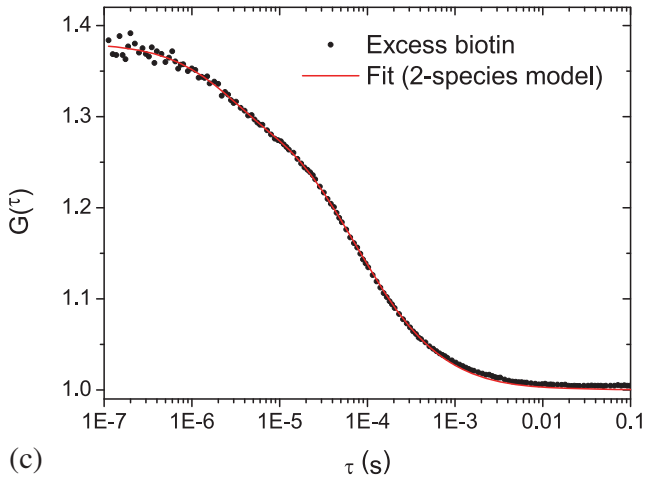

Fig. 4. Fluorescence correlation measurements and fitting curves according to the models discussed in the text for: (a) free Biotin; (b) Biotin, in the presence of an excess of Streptavidin; (c) Streptavidin, with an excess of Biotin. Across all samples, Biotin was kept at a concentration of $100 \mathrm{nM}$.

\section{Results and discussion}

\subsection{FCS measurements}

The FCS data from the three samples (free Biotin, Biotin with excess Streptavidin, and Streptavidin with excess Biotin) are shown in Fig. 4(a)-Fig. 4(c) and the corresponding fit parameters are summarized in Table 1.

From the one-species fit of the free Biotin data (Fig. 4(a)), we extracted the diffusion time of Biotin, $\tau_{D, B}$. Namely, $\tau_{D, B}=50 \pm 1 \mu \mathrm{s}$. The triplet amplitude, $T$, 
Table 1. FCS data.

\begin{tabular}{lcccccc}
\hline & $N$ & $m$ & $\tau_{D, B}[\mu \mathrm{s}]$ & $\tau_{D, S}[\mu \mathrm{s}]$ & $T$ & $\tau_{T}[\mu \mathrm{s}]$ \\
\hline Biotin & $4.0 \pm 0.4$ & 1 (fixed) & $50 \pm 1$ & & $0.348 \pm 0.002$ & $1.67 \pm 0.06$ \\
Excess Strept. & $4.6 \pm 0.5$ & 0 (fixed) & & $190 \pm 30$ & $0.36 \pm 0.08$ & $3.1 \pm 0.4$ \\
Excess Biotin & $3.6 \pm 0.3$ & $0.42 \pm 0.08$ & 50 (fixed) & 190 (fixed) & $0.180 \pm 0.004$ & $2.3 \pm 0.1$ \\
\hline
\end{tabular}

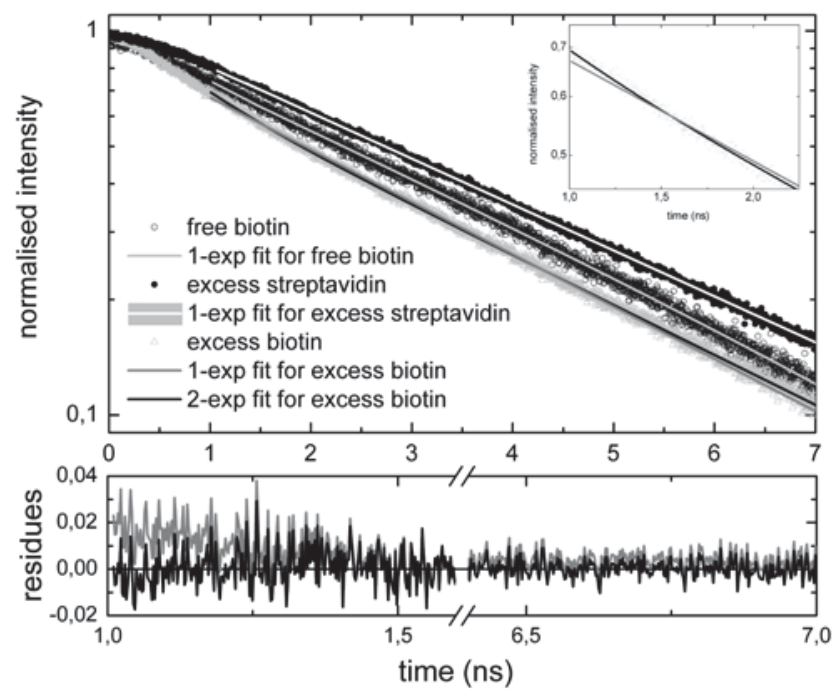

Fig. 5. Upper panel: time-correlated single photon counting data. Inset: expansion of the decay distribution for excess Biotin shortly after excitation, where 1-exp and 2-exp models are maximally different. Lower panel: residues for the excess-Biotin patters fitted to 1-exp (gray line) and 2-exp (black line) models.

and the triplet lifetime $\tau_{T}$ were also assessed by the fitting, and they resulted to be $T=0.348 \pm 0.002$ and $\tau_{T}=1.67 \pm 0.06 \mu \mathrm{s}$, respectively. The above values represent the average on the fits corresponding to three different datasets, with uncertainties given by the corresponding standard deviations. The same approach was used to get the diffusion time for Streptavidin, $\tau_{D, S}$. The data for Biotin with an excess of Streptavidin (Fig. 4(b)) was also fitted with the one-species model. This assumes that only bound Biotin is observed, an assumption that is also supported by the lifetime measurements described below. We found a diffusion time of $\tau_{D, S}=190 \pm 30 \mu \mathrm{s}$, a triplet amplitude of $0.36 \pm 0.08$ and a triplet lifetime of $3.1 \pm 0.4 \mu \mathrm{s}$.

In the last sample, containing Streptavidin with an excess of Biotin, we assumed the presence of two species: free Biotin and saturated Streptavidin. This is again corroborated by the lifetime measurements presented below. We fitted the data (fig. 4(c)) with the two-species model (Eq. (20)), fixing $\tau_{D, B}(50 \mu \mathrm{s})$ and $\tau_{D, S}(190 \mu \mathrm{s})$. The resulting fit gave a consistent triplet lifetime $(2.3 \pm 0.1 \mu \mathrm{s})$, and a slightly less consistent triplet amplitude $(0.180 \pm 0.004)$. It should be noted that fitting the one-species model to this data did not produce a good fit (data not shown).

\subsection{TCSPC measurements}

The above described FCS experiment was coupled to a TCSPC analysis performed on three solutions with Biotin to Streptavidin concentration ratios representing the same extreme instances of the binding equilibrium. The TCSPC data from the three samples are shown in fig. 5 and the corresponding fitting parameters are summarized 
Table 2. TCSPC data.

\begin{tabular}{lcccccc}
\hline & $\tau_{\mathbf{I}}[\mathrm{ns}]$ & $f_{\mathbf{I}}$ & $\tau_{\mathbf{I I}}[\mathrm{ns}]$ & $f_{\mathbf{I I}}$ & $\tau_{\mathbf{I I I}}[\mathrm{ns}]$ & $f_{\mathbf{I I I}}$ \\
\hline Biotin & & & & & $3.35 \pm 0.02$ & 1 \\
Excess Strept. & & & $3.60 \pm 0.02$ & 1 & & \\
Excess Biotin & $0.60 \pm 0.01$ & $0.47 \pm 0.02$ & & & $3.34 \pm 0.02$ & $0.53 \pm 0.02$ \\
\hline
\end{tabular}

in Table 2. The first solution contained only Biotin. It could be optimally fitted by a single exponential decay model, with a lifetime value of $3.35 \pm 0.02 \mathrm{~ns}$, which is comparable to the lifetime of free Atto- 550 in water as indicated by the producer, 3.6 ns [13]. A second solution featured an excess of Streptavidin. In this case, virtually all the Biotin molecules are expected to be bound to Streptavidin molecules and fluorescence to be essentially emitted by 1:1 Biotin-Streptavidin complexes (species II in Fig. 1). Once again, we managed to fit the experimental decay with a single decay component, whose lifetime was slightly longer than that measured for free Biotin, namely $3.60 \pm 0.02 \mathrm{~ns}$. The third instance we investigated was a solution displaying an excess of Biotin. The solution mainly contained free Biotin and saturated 4:1 Biotin-Streptavidin complexes. Notably, the experimental decay pattern obtained for this solution displayed two different decay components (see Fig. 5). One component decayed with a lifetime equal to that measured in the case of free Biotin within experimental error $(3.34 \pm 0.02 \mathrm{~ns})$. The other one, that we attribute to the decay of saturated 4:1 Biotin-Streptavidin complexes, had the considerably shorter lifetime of $0.60 \pm 0.01 \mathrm{~ns}$. Such a shortening of the Atto-550 dye lifetime upon formation of the 4:1 complex can be explained by taking into account the self-quenching of the fluorophores. The Atto-550-Biotin molecules that are bound to one Streptavidin are separated by distances [16] smaller than the Förster radius [13]; this opens up a non-radiative decay pathway due to FRET.

\subsection{Discussion}

Although additional calibrations would be needed in order to calculate absolute diffusion constants, we can understand the ratio between the diffusion times of Biotin and Streptavidin. From the Einstein-Stokes relation [17], we can easily derive that:

$$
\frac{\tau_{D, B}}{\tau_{D, S}}=\frac{D_{B}}{D_{S}}=\frac{R_{S}}{R_{B}}=\sqrt[3]{\frac{V_{S}}{V_{B}}} .
$$

Assuming equal densities for the Biotin and Streptavidin molecules, we find that:

$$
\frac{\tau_{D, B}}{\tau_{D, S}}=\sqrt[3]{\frac{m_{B}}{m_{S}}} .
$$

This prediction holds true within 1.5\%: $\tau_{D, B} / \tau_{D, S} \sim 0.263$ and $\sqrt[3]{m_{B} / m_{S}} \sim 0.267$.

Analysis of the two-species data is challenging. While we could fit the data in Fig. 4(c) using the Biotin and Streptavidin diffusion times obtained earlier, the fit allows for a much wider range of diffusion times. This limits the value of the result. Additionally, if one were to extract meaningful information about the relative population sizes of Biotin and Streptavidin, more precise control of the sample concentrations would be needed. Moreover, setting the value of $\eta$-parameter to 1 might in principle affect the determination of the $m$-value (see Eq. (20)). In fact, the $\eta, N$, and $m$ parameters are strongly coupled one to the other, and consequently the determination of 
the three parameters separately without any a-priori knowledge of the system would be both very difficult and scarcely reliable. Nevertheless, such an approximation is reasonable as:

- in the assumption that the radiative decay constant of Atto-550 does not change upon binding of biotin to streptavidin, the ratios of the decay times determined in the TCSPC experiment are indicative of the relative quantum yields of Atto-550 molecules labelling species I, II, and III. Provided that also the absorption cross section of I, II, and III is similar, as $\tau_{I I I}=3.35 \mathrm{~ns}$ and $\tau_{I I}=3.6 \mathrm{~ns}, \eta \sim 0.93$. As to species I $\left(\tau_{I}=0.6 \mathrm{~ns}\right)$, the molecular brightness of a single Atto- 550 molecule would be 5.6 times smaller than that of species III. However, as four molecules of dye would be bound to each saturated Streptavidin molecule, $\eta \sim 4 / 5.6=0.72$, a value that is not so different from 1 .

- The $N$ value determined in the case of excess Biotin roughly agrees with those found for the biotin and excess Streptavidin samples, whose overall biotin concentration is equal. Moreover the free-Biotin fraction value ( $m$ in Eq. (20)) is comparable to the free-Biotin fraction derived from TCSPC data for the same sample. The above correspondences suggest that the $\eta$ value should not be very different from 1.

The TCSPC measurements demonstrate that two different species coexist in the excess Biotin sample, which should be free Biotin and saturated Streptavidin. However, due to the small difference in lifetime between free Biotin and 1:1 Biotin-Streptavidin complex (species II), we cannot rule out the possibility that also traces of the latter species are present in this instance. Conversely, there is a clear difference in fluorescence lifetimes between the saturated Streptavidin complex and free Biotin. Namely, the former manifested a lifetime being six times shorter than the latter. If the difference is totally attributed to FRET, a mean interchromophore distance of the order of $3 / 4 R_{0}$ [13] is inferred, which is notably in agreement with crystallography data [16]. The slightly longer lifetime manifested by Atto-550 in the 1:1 BiotinStreptavidin complex might be due to the partial shielding of the fluorophore in the binding site from interaction with the solvent molecules.

\section{Conclusions}

In the present research we have performed preliminary experiments on the BiotinStreptavidin system, using a combination of fluorescence correlation spectroscopy and time-correlated single photon counting measurements. Using fluorescence correlation spectroscopy, we have been able to extract relative diffusion coefficients for free and Streptavidin-bound Biotin; their ratio finely matches theoretical predictions. Timecorrelated single-photon counting indicated the presence of three different species in the considered solutions and pointed out major differences in the corresponding lifetimes. This work can be considered as a starting point for further investigations of Biotin-Streptavidin binding dynamics, which will be devoted to clarify whether the binding is cooperative or not.

The experiment has been performed in the context of STELLA, the School for Training in Experiments with Lasers and Laser Applications, hold at the Insubria University in Como from June 20 to July 8, 2011 (see www. stella-school .eu). The Authors wish to acknowledge CARIPLO, UNIVERCOMO and Banca del Monte di Lombardia Foundations for having financed the project. The Authors like also to acknowledge the STELLA-school students T. Makoto, N. Mueller, and K. Spasibko for their help in experimental work and A. Andreoni for fruitful discussion. 


\section{References}

1. A. Holmberg, A. Blomstergren, O. Nord, M. Lukacs, J. Lundeberg, M. Uhlen, Electrophoresis 26, 501 (2005)

2. K. Svoboda, S.M. Block, Annu. Rev. Biophys. Biomol. Struct. 23, 247 (1994)

3. S.B. Smith, Y. Cui, C. Bustamante, Science 271, 795 (1996)

4. H. Kim, I.-H. Cho, J.H. Park, S. Kim, S.-H. Paek, J. Noh, H. Lee, Colloids and Surfaces A: Physicochem. Eng. Aspects 313-314, 541 (2007)

5. M. Lenhert, M. Gorbahn, C. Rosin, M. Klein, I. Köper, B. Al-Nawas, W. Knoll, M. Veith, Langmuir 27, 7743 (2011)

6. M. Gonzales, C.E. Argaraña, G.D. Fidelio, Biomol. Eng. 16, 67 (1999)

7. T. Sano, C.R. Cantor, J. Biol. Chem. 265, 3369 (1990)

8. M.L. Jones, G.P. Kurzban, Biochem. 34, 11750 (1995)

9. A. Loosli, U.E. Rusbandi, J. Gradinaru, K. Bernauer, C.W. Schlaepfer, M. Meyer, S. Mazurek, M. Novic, T.R. Ward, Inorg. Chem. 45, 660 (2006)

10. R. Rigler, U. Mets, J. Widengren, P. Kask, Eur. Biophys. J. 22, 169 (1993)

11. P. Schwille, Cell Biochem. Biophys. 34, 283 (2001)

12. M. Goesch, R. Rigler, Adv. Drug Deliv. Rev. 57, 169 (2005)

13. ATTO-TEC 2011 Online Catalogue: http://www.atto-tec.com/index.php?id=54 no_cache $=1 \backslash \mathrm{L}=1$

14. A. Andreoni, M. Bondani, L. Nardo, Mol. Cell. Probes 23, 119 (2009)

15. W. Becker, The bh TCSPC Handbook - Third Edition (Becker \& Hickl, GmbH, 2008), p. 401

16. S. Freitag, I. Le Trang, L. Klumb, P.S. Stayton, R.E. Stenkamp, Prot. Sci. 6, 1157 (1997)

17. A. Einstein, Ann. der Physik 17, 549 (1905) 